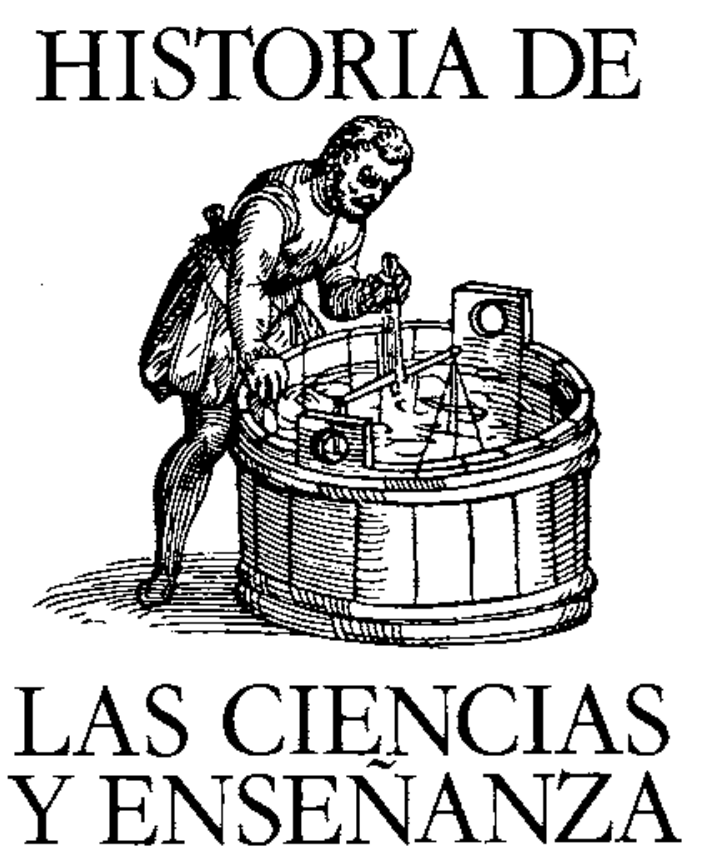

\title{
USOS Y ABUSOS DE LA HISTORIA DE LA FÍSICA EN LA ENSENAANZA
}

\section{SÁNCHEZ RON, J.M. \\ Departamento de Física Teórica. Universidad Autónoma de Madrid.}

Conferencia invitada en el II Congreso Internacional sobre Investigación en la Didáctica de las Ciencias y las Matemáticas. Valencia 23-25 de septiembre de 1987.

\section{SUMMARY}

This paper contributes to the debate concerning the introduction of the History of Science -more specifically the History of Physics- into Secondary Education and University curricula.

\section{INTRODUCCIÓN}

Desde hace ya más de veinte años la historia de la ciencia y la historia de la física en particular viene experimentando un auge considerable, (1) tanto en número y calidad de investigadores, como en la recepción que los trabajos de éstos tienen entre el gran público o, dejémoslo en esto, entre aquellos con ciertos intereses intelectuales. La ocasión es, por otra parte, enormemente propicia; vivimos en un perfodo histórico en el que los valores cientifico-tecnológicos han adquirido una indudable popularidad. Es la era de las «nuevas tecnologías». Hiroshima y más tarde la crisis del petróleo de los años 70 vinieron a recordarnos cuán poco fiables son los elementos naturales (sea este soldado o crudo petrolífero accesible y barato). Los ciudadanos del último tercio del siglo $\mathrm{XX}$ tienden, mayoritariamente, a pensar que la ciencia resolverá todos sus probiemas. 
Ante esta situación no tiene mucho de sorprendente el que se haya abierto un dcbate -todavía inconclusorelativo a la conveniencia de incluir o no la historia de la ciencia y/o las historias de las distintas ciencias en la enscĩanza subuniversitaria o en las carreras universitarias. Mi contribución en esta ocasión se centrará en este tema, basándome en lo que es mi especialidad, la historia de la física, y con el escaso bagaje de seis años de experiencia como profesor de la asignatura (optativa) de historia de la física en 3er. curso de la licenciatura en Ciencias Físicas de la Universidad Autónoma de Madrid. Contémplese, por consiguiente, mi discusión como una aportación teórica, especulativa, a esta importante cuestión.

\section{2. ¿POR QUÉ HISTORIA DE LA FÍSICA?}

Un primer problema con el título que he elegido es el de por qué restringirse a la historia de la física. Naturalmente, siempre podría decir, como he apuntado hace un instante, que el motivo se encuentra en que esa es mi especialidad, y que uno habla, o debe hacerlo, de lo que sabe. Pero, aunque esto es cicrto, hay algo más detrás de semejante restricción. Me explicaré.

Aunque la situación ha cambiado substancialmente en los últimos tiempos, cs el nuestro un siglo en el que han predominado, de manera abrumadora, los descubrimientos físicos, algunos de ellos, como la relatividad y la mecánica cuántica, auténticamente espectaculares. Estos logros desataron fuerzas, apocalípticas unas, liberadoras otras, sin las cuales no es posible entender la sociedad mundial actual. La energía atómica, el vertiginoso desarrollo experimentado en la transmisión de comunicaciones (transistores, chips, fibra óptica), o los nuevos materiales, avalan la opinión de que vivimos en el «siglo de la Física». Más aún, algunos de los descubrimientos científicos más importantes realizados en este siglo fuera del dominio de la Física, han dependi* do de diversas mancras, de esta disciplina. Así ocurre, por ejemplo, con la teoría del desplazamiento de los continentes, como se puede ver tanto en el hecho de que su promotor, Alfred Wegener, fuese de formación astrónomo, geofísico y meteórologo, como en sus esfucrzos por encontrar un mecanismo físico que pudiese explicar la movilidad de los continentes. Todavía de manera más dramática - y decisiva- aparece en la intervención del paleomagnetismo en la confirmación de la teoría tectónica de placas, el actual marco teórico del desplazamiento continental. De manera análoga, la Física y algunos físicos (Schröedinger, Srilard, Delbrück) fueron elementos importantes en el descubrimiento de la estructura del ADN, la molécula transmisora de la herencia. Sin la Física moderna, sin la difracción de rayos $\mathrm{X}$ en concreto, dificilmente podría existir la Biología Molecular.

En otras palabras, si -supongámoslo así por un momento- se cree conveniente inclutr a la historia de la ciencia dentro de los programas de estudio, y si se acepta que uno de los propósitos que debe guiar a estos es el de ayudar a los estudiantes a comprender su entorno, físico, social e intelectual, más próximo, cntonces parece difícil negar que la historia de la fístca debe jugar un papel «especial», nunca exclusivo o ex. cluyente, en el diseño de tales programas.

De hecho, la importancia de la física para las demás ciencias es algo que se puede retrotraer cuando menos al siglo XVII. La publicación, en 1687, de los Philosophiae naturalis principia matematica de Isaac Newton abrió una era en la que la física se constituyó en modelo y herramienta para el desarrollo de la mayoría de las ciencias, desde la química a la medicina, sin olvidar algunas ramas de las humanidades. En efecto, la filosofía natural newtoniana afectó profundamente al pensamiento social y político, a las ideas relativas a la religión, c incluso al arte. Montesquieu escribió acerca de la gravitación universal newtoniana o «poder de gravitación» en su exposición del «principio de Monarquía» en su Esprit des Lois y John Adams invocó la tercera ley de movimiento de Newton para defender la nueva Constitución de los Estados Unidos. Como ejemplo final se puede recordar a J.T. Desaguliers, que escribió una obra política titulada El sistema rewtoniano del mundo el mejor modelo de gobierno.

Entramos de esta manera en el «uso» -al que no lc falta, como veremos más adelante, su correspondiente «abuso»- de la historia de la física en la enseñanza. Exploremos de manera más detenida algunas de sus implicaciones.

\section{HISTORIA DE LA CIENCIA VERSUS HIS- TORIA GENERAL}

Es bien sabido - la filosofía, la metodología en concreto, te la ciencia ha popularizado esta distincion-mque la historia de la ciencia se puede dividir en, al menos, dos categorias: historia interna e historia externa; en la primera son las ideas, teorias, experimentos, el contenido en definitiva de la ciencia en consideración el objeto primario, mientras que en la segunda, que también se podria denominar historia social, o económica, social y politica de, por supuesto, la ciencia, lo principal son todos Ios condicionantes o circunstancias, que rodean a la actividad científica. Obviamente, para poder llevar a la práctica el que, como decía antes, los programas de estudio estén diseñados para ayudar a los estudiantes a comprender su entorno, física, social $c$ intelectual, es preciso recurrir a la historia interna cuando se trate del entorno físico, a la externa para el social, y a una mezcla de ambas en el caso del entorno intelectual.

La pregunta que ahora surge de forma natural es la siguiente: $i$ debemos dar preferencia a alguno de los dos tipos de historia de la ciencia, la internalista o la exter. 
nalista, en la enserranza? Desde mi punto de vista no existe una contestación general a esta pregunta, una res. puesta válida independiente del tipo de enseñanza, y para lo que sigue dividiré a ésta, un tanto groseramente, en tres grupos: el de la enseñanza subuniversitaria, el de la universitaria y el de la normal.

En lo relativo al nivel universitario, que discutiré de manera independiente más adelante, diré, adelantan. do ideas, que la situación no es la misma para los dife. rentes estudios. Considero importante introducir asignaturas de historia de la ciencia, basadas en los aspectos externalistas, socieconómicos, en carreras como las de Filosofía, Historia o Económicas. Para las carreras cientifficas (Facultades) o técnicas (Escuelas de Ingenieros) mis dudas son considerables. Dicho de otra manera, si lo que se pretende es formar buenos profesio. nales, no se ha demostrado que la historia de la ciencia, general o particular, sea necesaria. Por el contra. rio, sí se ha argumentado que puede ser perjudicial. Pero sobre esto, más adelante.

Paso, por consiguiente, al nivel subuniversitario, al que ahora se denominaba «bachillerato superior». Aquí nos encontramos con dos parcelas en mi opinión perfectamente diferenciadas. La primera se refiere al papel de la historia de las ciencias en la enseñanza de las ciencias, pero esta cuestión la discutiré, en el caso de la física, en la próxima sección. Mi intención ahora es centrarme, tal y como exige el titulo de este apartado («Historia social versus historial general»), en el del papel de la historia de las ciencias en la enseñanza de las materias no científicas, en la enseñanza, en particular, de los temas históricos y sociales. Ya me he referido, de manera breve e incompleta, a la importancia de la ciencia en los acontecimientos socioeconómicos y políticos, especialmente de nuestro siglo (no habria ninguna difícultad en extender la discusión a otros periodos, at menos a partir de los siglos XVI y XVII), pero que algo sea importante no quiere decir que debamos enseñarlo a jóvencs de, en este caso, 15, 16017 años aproximadamente.

No es éste, sin embargo, el caso. No existe, en mi opinión, nada que impida o desaconseje la incorporación de la historia de la ciencia en las asignaturas históricosociales del bachillerato y sí mucho que lo recomienda.

En primer lugar tememos que al estar tratando con la ciencia como un fenómeno, como una actividad, social se minimizan las dificultades de comprensión por parte de unos jóvenes con un conocimiento muy somero de las disciplinas científicas (en el mejor de los casos). Son muchos los ejemplos que se pueden ofrecer en este sentido; pondré algunos: Las relaciones políticas entre Gran Bretaña y Alemania a lo largo del siglo XIX y primera mitad del XX han sido, como se sabe, complejas y su conocimiento imprescindible para cualquier historia contemporánea; pues bien, dificilmente se obtendrá una imagen adecuada de ellas sin tomar en consideración como se percibian en ambos países los respectivos avances o, simplemente, potencia científico-tecnológica y educativa ${ }^{(2)}$.

Un segundo posible ejemplo lo encontramos en la revolución científica de los siglos XVI y XVII. Al margen de una presentación de las ideas y teorías de los Kepler, Galileo, Gilbert, Brahe, Descartes o Newton, es posible, recomendable y no excesivamente complicado (accesible desde luego a un joven no universitario más fácilmente que las doctrinas de Kant o Hegel), discutir estos siglos que dieron lugar al conocimiento de la ciencia moderna haciendo hincapié en la aparición de socicdades científicas como las Accademia dei Lincei, Accademia del Cimento, Royal Society, Académie des Sciences, Societas treunetica, Collegium Naturae Curiosum o la Academia de Ciencias de Berlín ${ }^{(3)}$, esto es, resaltando la componente colectiva, asociativa, de la ciencia. Otra faceta a la que se debería prestar atención al hablar de este periodo es la de la renovación metodológica defendida y practicada entonces. Francis Bacon, con su Novum organum (1620), Descartes o Newton son, evidentemente, algunos de los casos a discutir en este sentido(4). Un tercer, y triviaI, posible tercer ejemplo es el de las repercusiones e incidencias sobre los descubrimientos realizados en física nuclear en la década de 1930. Tratando este tema el profesor de «historia general» puede introducir, o desarrollar, cuestiones tan interesantes - y tan diversasde la historia contemporánea como la política racista introducida por Hitler en abril de 1933, el exilio masivo de intelectuales judios centroeuropeos, el desplazamiento del liderazgo cientifico y tecnológico de Europa a Estados Unidos, el proyecto Manhattan, la conclusión de la Segunda Guerra Mundial, la guerra fria y la política energética, sus posibilidades y problemas (movilizaciones populares entre ellos), de la segunda mitad de nuestro siglo.

Las ventajas asociadas con la introducción de la histo. ria de la ciencia en la "historia general» son numerosos. Por un lado está el mejorar la imagen de la historia que se ofrece a los estudiantes. La ciencia y la tecnologia han sido dos instrumentos esenciales en el progreso de la humanidad y su exclusión en cualquier intento de reconstrucción histórica reduce considerablemente sus pretensiones. Pero además está el que la historia no sólo tiene como función el comprender el pasado (y el presente) sino también el prepararse para afrontar el futuro (que siempre empieza «justo a continuación) ), y si, como comencé diciendo, vivimos en una era, la de las «nuevas tecnologias», en la que el sistema ciencia-tecnologia es uno de los valores sociales más cotizados y admirados, cuyo declive no parece vislumbrarse en el futuro proximo, entonces la introducción de la historia de la ciencia en las disciplinas socio-históricas estudiadas por los jovenes se convierte casi en un deber social. El monstruo no nos devorará si lo conocemos.

Existen varios problemas para lievar a la práctica las ideas que acabo de esbozar. El principal es que la ta- 
rea de insertar la historia de la ciencia en la «historia general» está todavía en sus inicios. Los «historiado. res generales» no han explotado en absoluto la ya considerable riqueza de trabajos producidos durante las dos últimas décadas por los historiadores de la ciencia. Sintesis generales de la historia mundial tan impresionantes como las de Toynbec, con su veterano Es. tudio de la historia, o Braudel (Civilización material, economía y capitalismo, siglos $X V-X V I I I)$ son muy pobres, tanto en lo relativo a rigor como en extensión, en sus tratamientos de los aspectos científicos.

¿Dónde, por consiguiente, debc buscar el profesor que quiere explicar un curso o que, acaso, desea escribir un libro de texto o un manual de apoyo de acuerdo con las lineas directrices anteriores? El problema es peliagudo; es posible sacar un poco de aquí y otro poco de allá rebuscando entre la maraña de libros existentes. Yo creo que es más una tarea de equipos que de individuos, una tarea en cualquier caso todavía por hacer y en la que no será trivial convencer al profesor de historia que debe ampliar las perspectivas tradicionales de su disciplina. Como punto de partida yo recomendaría las siguientes obras, en las que, justo es decirlo, la física resulta particularmente beneficiàda: La Historia social de la ciencia ${ }^{(5)}$ de John D. Bernal, aún útil a pesar de sus manifiestas limitaciones (corsé ideológico -el marxismo de Bernai- así como la escasa aitura y fiabilidad de sus investigaciones o manifestaciones historicas), The scientist' role in society. A comparative study ${ }^{(6)}$ de Joseph Ben-David, Science and social change, $\left.1700-1900^{\circ}\right)$ de Colin Russelt, Ciencia, tecno. logia y sociedad en la Inglaterra del siglo $X V I I^{(8)}$ de Robert K. Merton, Science and Society ${ }^{(9)}$ de Hilary y Steven Rose, Physics and its fifth dimension: Society ${ }^{(10)}$ de Dietrich Schroeer, La sociologia de la ciencia ${ }^{(1)}$ de Robert $\mathrm{K}$. Merton y, en un plan más modesto y divulgador, La ciencia europea del siglo $X X^{(12)}$. Más especializados pero modélicos en sus respectivos temas son las obras de A. Hunter Dupree, Science in the Federal Governmend ${ }^{13)}$, Alan D. Beyerchen, Scientists under Hitler. Politics and the Physicists Community in the Fhird Reich ${ }^{(14)}$, y Lewis Pyen. son, Cultural Imperialism and the Exact Sciences. German Expansion Overseas, 1900-1930(15). Finalmente señalar que es posible extraer una información bastante útil e interesante de experiencias de cursos de historia social de la ciencia, como el que desde 1972 se viene desarrollando en la «Unidad de Estudios Científicos» de la Universidad de Edimburgo, aunque está diseña. do para estudiantes de Facultades de Ciencias. Steven Shapin describió el curso hace algunos años en la revista Social Studies of Science ${ }^{(16)}$ a la que remito a todos los interesados.

Un último comentario antes de finalizar esta sección. La inclusión de la historia de la ciencia dentro de los programas socio-históricos no redundará únicamente en un mejor conocimiento, por parte de los estudiantes, de la realidad histórica; también mejorará, o co* menzará a formarse, su idea de la actividad cientifica, de lo qué es la ciencia, algo sin duda beneficioso para los futuros aprendices - universitarios-de científicos. La formación, de la que hablaré más adelante, en metodología de la ciencia se verá de esta manera complementada y facilitada.

\section{HISTORIA DE LA FÍSICA Y ENSEÑANZA (NO UNIVERSITARIA) DE LAS CIENCIAS}

Existen dos posibles formas de justificar la enseñanza de la historia de ia ciencia, al margen de su importancia -ya discutida - para comprender mejor la "his toria general»s. La primera justificación es independien* te de cualquier otra asignatura. La ciencia es una actividad humana importante, una de las caracteristicas, de hecho, que distinguen a la especie humana de los animales, y por consiguiente merecedora de que se estudie su historia, la evolución de sus ideas, experimentos, teorias, científicos, instituciones y repercusiones. No es dificil argumentar que la historia de la ciencia es más conveniente para un hombre o mujer medianamente educado que la historia de la filosofia o la historia del arte, y si ésta es así no sería descabellado incluirla, de manera autónoma, en los programas de bachillerato $\rightarrow$ como parece que va a ocurrir $\rightarrow$ salvo que lo impida el número de otras asignaturas igual o más importantes.

Pero yo no pretendo insistir aquí en esta posible justificación, sino en otra mucho menos evidente, más pro. blemática, $\mathrm{y}$, no obstante, para algunas cuestiones, más transcendente. Me estoy refiriendo al papel de la historia de la ciencia en la enseñanza de la ciencia.

A menudo se ha esgrimido en favor de las asignaturas de historia de la ciencia o, mejor, del uso de la historia de la ciencia en las asignaturas científicas, el que permiten comprender mejor la propia ciencia. Sobre este punto, auténticamente crucial, diré de entrada que no está demostrado en absoluto y que, además, en mi opinión, es tomado en general y sin más falso. Ahora bien, cuando digo "falso", quiero decir, repito, falso en general, en cualquier circunstancia, y no que no sea posible o conveniente utilizar, de forma controlada, la his. toria de la ciencia para, efectivamente, facilitar la comprensión del contenido o alcance de ideas científicas.

El principal problema reside en que una historia rigurosa -lo que no quiere decir «no simplificada» de la ciencia no permite descubrir la existencia de un método científico. Varias décadas de continuo debate metodológico han conducido al estado de opinión actual según el cual las recetas propuestas por el positivismo lógico, Popper, Kuhn, Lakatos, Toulmin o Laudan son atractivas pero atractivamente erroneas. Hoy estamos más cerca del anarquismo metodológico de Fcyerabend o del sociologismo de Merton y de la Solla Price que de aquellos. (Esto no quiere decir, sin embargo, que 
no sea conveniente incluir la «metodología de la ciencia» dentro de los programas de bachillerato - probablemente como parte de la asignatura de filosofia- todo lo contrario, la metodologia científica no nos habria enseñado qué es la ciencia, pero sí que ha contri. buido a aclaramos algunas de las cosas que no es la ciencia, lo que no deja de ser una lección francamente saludable y necesaria).

Y si no existe método, si no hay una receta que descubrir como "hacer ciencia» ¿para qué querrán la historia de la ciencia los profesores de ciencias, curiosidad aparte? Valdrá sin duda para demostrar que «los caminos del descubrimiento científico son inescrutables», para valorar el elemento creativo, anárquico o heterodoxo que, moderada y ocasionalmente, caracteriza a la ciencia. Ahora bien si entramos por ejemplo en la cuestión del «descubrimiento científico versus enseñanza por descubrimiento", en el llamado «aprendizaje por descubrimiento" asociado a los nombres de Dewey y Bruner, entonces el papel de la historia de la ciencia se hace más cuestionable. Para ver que esto es así tomemos una de las características que, de acuerdo con Gené y Gil(17), tiene una enseñanza por descubrimiento. «Una enseñanza por descubrimiento... debería venir caracterizada", escriben estos autores (p. 65), por «una traducción de la información, que habitualmente se proporciona elaborada, a problemas que permitan rehacer en cierto modo el proceso histórico. Problemas por otra parte que deben ser presentados de forma ordenada, con un claro hilo conductor, evitando adquisiciones dispersas y proporcionando una visión coherente).

Nadie negará que, tal y como indican Gené y Gil, para enseñar descubriendo, la claridad, el orden, los hilos conductores son requisitos casi imprescindibles. Ante una variedad de opciones igualmente plausibles, confrontado con una falta de necesidad lógica, el estudiante se pierde, se desconcierta, y el problema es que cuanto más vamos sabiendo sobre la historia de fa ciencia, de la física al menos, más pluralidad, menos «necesidad» nos encontramos. El proceso de creación científica, el denominado "contexto de descubrimiento", es una delicada flor, un complicado laberinto en el que se entrecruzan todo tipo de motivaciones e intuiciones, respetando, eso sí, el principio de una eventual (que puede estar lejos en el futuro) contrastación con la experiencia (contrastación, como es sabido, muy problemática también desde su punto de vista metodológico; cuestión de "términos teóricos» versus "términos ex" perimentales», o imposibilidad de refutación estricta de una teoria científica). Algunos ejemplos pueden ser apropiados: Las investigaciones de Oersted en electri. cidad estuvieron motivadas por su creencia metafísica en la unidad de las fuerzas de la naturaleza, a la que llegó bajo la influencia de ia Naturphilosophie dominante en Alemania a comienzos del siglo XIX y cuyo principal exponente fue F.W.J. Schelling. Kepler creía firmemente en la "música de las esferas» que propug- nó Pitágoras y sus seguidores, tratando incluso de averiguar las notas que cada planeta emitía en su movimiento; la tres leyes keplerianas del movimiento planetario no son ajenas a tales intereses. Planck para quien la búsqueda de lo absoluto era el fin último de toda ciencia. Mach quien por el contrario defendía la imposibilidad de ir más allá de las sensaciones, lo que le alejaba del absoluto planckiano. Leibniz basando su física en los principios de razón suficiente y de identidad de los indiscernibles. Newton defendiendo su «hypotheses nou fingo». Dirac aceptando como criterio heurístico de verdad física la belieza matemática. Heisenberg primando lo observable, lo discontinuo, en su mecánica de matrices. Schrödinger rechazando los puntos de vista de Heisenberg, y dando primacía al continuo en su mecánica ondulatoria. O Einstein desdenando las observaciones experimentales de Kauffman que contradecían su teoria de la relatividad espacial.

La situación no es, sin embargo, tan desesperada (para la historia de la ciencia) como podría parecer. No, al menos, al nivel del bachillerato. Existen niveles educativos en los que, creo yo, enseñar es mentir un poco. Entiéndaseme lo que quiero decir. Enseñar es con frecuencia elegir, simplificar. No parece razonable ser excesivamente preciso en el bachillerato, por ejemplo. El que la electrostática no sea, eventualmente, más que un caso extremadamente restringido de la electrodinámica cuántica no impide que figure en nuestros textos, que, muy razonablemente, no mencionan siquiera su versión cuántica. De manera análoga no debe repugnar eí seleccionar ejemplos históricos particularmente convenientes para ilustrar algunos puntos importantes de tal o cual ciencia. Recordemos en este sentido las palabras de Gené y Gil: «Una enseñanza por descubrimicnto... deberfa venir caracterizada... Por una «traducción» de la información, que habitualmente se proporciona elaborada, a problemas que permitan rehacer en cierto modo el proceso histórico (énfasis añadido). El «en cierto modo» no es superfluo.

Es importante, desde esta perspectiva, el seleccionar ejemplos que se puedan insertar en los libros de texto de ciencias para ilustrar algún punto de interés. Esto es lo que haré a continuación con algunos ejemplos relativos a la física, no sin antes señalar que en mi opinión esta es una de las tareas más importantes a realizar por profesores e investigadores no ajenos a los problemas didácticos del bachilierato.

(a) Galileo y la confirmación del sistema heliocéntri. co, o la importancia de los instrumentos en la física.

Por muy importante que sea la teoría para la física, ésta no existiría sin el experimento, sin el contacto, controlado, con la naturaleza. Las dificultades de todo ti. po inherentes en la instalación de buenos laboratorios, hace que se caiga en múltiples ocasiones en una enseñanza excesivamente libresca y teórica de la física. Es necesario, por consiguiente, recalcar en los libros de texto de fística la importancia de la experimentación y 
a falta de bucnos laboratorios la historia de la física puede ayudar. Un ejemplo fácilmente documentable lo tenemos en Galileo y el telescopio. Sólo después de que Galileo construyese un telescopio (primero de 8 aumentos, meses después de 20 ) en el verano de 1608 pudo embarcarse en una serie de observaciones que le permitieron defender ante el grueso de la comunidad científica su copernicanismo previo. Los descubrimientos astronómicos que, a partir de 1609 , Galileo efectuó con sus telescopios, el relieve de la Luna, los satélites de Jupiter, las manchas del Sol y las fases de Venus, harian posible, a pesar de todos los problemas que la Iglesia romana, la ortodoxia aristotélico-ptolemaica o, simplemente, la inercia de pensamiento impusieron el que sistema geocéntrico (ptolemaico) dejará sitio al heliocéntrico (copernicano) ${ }^{(18)}$.

La importancia de los aparatos para el desarrollo de la física también se puede resaltar mediante una discusión histórica de la interrelación existente entre el aumento de potencia ( $\mathrm{y}$ tamaño) de los aceleradores de partículas y el descubrimiento de nuevas partículas elementales, $o$, incluso, con una exposición del papel decisivo de los aparatos empleados por James Prescott Joule en la medida del equivalente mecánico del calor, descubrimiento clave para la formulación del principio de la conservación de la energia.

\section{(b) El descubrimiento newtoniano de la gravitación}

Isaac Newton figura, junto a Albert Einstein, en un lugar destacado dentro de los creadores de la ciencia moderna. Introducir, por consiguiente, a los estudiantes de bachillerato en algunos aspectos de su pensamiento y características de sus contribuciones es algo que no puede sino perdurar en un mejor conocimiento de la f́́sica básica. En el caso de Newton, y de Ios Principios en particular, somos afortunados al disponer de un artículo de I. Bernard Cohen en el que se discute con sencillez pero con profundidad al mismo tiempo, ese momento culminante de la Revolución Científica que fue el descubrimiento por parte de Newton de la ley de la gravitación universal(19). Las discusiones de Cohen de cómo Newton dedujo la naturaleza cuadrático-inversa de la fuerza centripeta (lo que es casi tanto como decir, la fuerza de atracción gravitacional) para el caso de órbitas circulares a partir de la tercera ley de Kepler y de la ley de la fuerza centripeta, o como esta última genera una trayectoria curva de acuerdo con la ley de las áreas son perfectamente asequibles para los estudiantes de un primer curso de física. Con estos ejercicios los alumnos no sólo se ejercitan en los rudimentos de la física sino que además adquieren un cierto conocimiento de los Principia, sin olvidar que también se ven introducidos, de la mano de Cohen, al denominado «estilo newtoniano», consistente en un continuo ir y venir entre un modelo matemático y la realidad física. (c) El papel de la noción de simetría en el descubrimiento einsteniano de la relatividad especial.

El cjemplo que voy a presentar a continuación puede ser utilizado en el último curso de física del bachillera. to -acaso antes - o en el curso de física general del primer año de universidad. Sería conveniente que el curso en cuestión incluyese una discusión de la relatividad especial, lo que, dicho sea de paso, es perfectamente asequible - aquí me baso en mi experiencia- a los estudiantes de los últimos años de bachillerato. Una manera simplificada de enseñar esta teoría einsteniana sería la siguiente: i) Introducir la noción de sistema iner. cial; ii) Formular la pregunta " ¿Cómo se relacionan las coordenadas de una particula medidas en dos sistemas inerciales diferentes? »; iii) Dar la respuesta de la mecánica newtoniana (transformaciones de Gaiileo). iv) Estudiar las consecuencias (principio de relatividad galileano, ley de transformación de velocidades); v) Presentar los problemas que llevaron a pensar que la ve. locidad de la tuz en el vacio es la misma en todos los sistemas inerciales, independientemente del movimiento relativo entre la fuente y el observador; vi) Enunciar los dos postulados de Einstein, vii) Introducir la relatividad del espacio y el tiempo como una consecuencia ineludible de los dos postulados anteriores; para la relatividad de la simultaneidad yo recomiendo el espléndido ejemplo utilizado por Gamow en su libro En el pais de las maravillas ${ }^{(20)}$ (pp. 75-78); viii) Escribir las transformaciones de Lorentz, y ix) Estudiar las conse. cuencias que se derivan de ella (contracción de longitudes, dilatación de tiempos y ley de composición de velocidades).

Pero dejemos este tema y volvamos a la cuestión que ahora nos ocupa. La física actual está basada en gran medida -me estoy refiriendo a sus aspectos más fundamentales- en la noción de simetría, de invariancia. La mecánica, ncwtoniana o telativista, la gravitación einsteniana, la mecánica cuántica, la física de la materia condensada, por no hablar de la física de partículas elementales, están empapadas de grupos de transformaciones, de simetrías, invariancias y leyes de conservación. No obstante, en los colegios y en los cursos de física general de las universidades seguimos enseñando la mecánica clásica y - cuando lo hacemos- la relativista, a la vieja usanza; esto es, sin hacer hincapié, sin emplear las técnicas y conceptos propios de las simetrías. Es preciso, por ejemplo, insistir en la existencia de un principio de "relatividad galileano» en la dinámica newtoniana. En otras palabras, hay que esforzarse por resaltar tanto la estructura formal común entre la mecánica clásica y la relativista, como las ventajas heurísticas que tiene el enfocar los problemas a la manera que ya defendiera, en otro contexto, Felix Klein con su «Programa de Erlangen» de 1872.

Ahora bien, las ventajas de la introducción de las si. metrías en la física se manifiestan sobre todo en sus niveles más avanzados, requieren, por consiguiente, un 
cierto grado de sofisticación (lo que no quiere decir que un tratamiento más moderno, a la manera que estoy promulgando aquí, de la mecánica en el bachillerato o primer curso de universidad no haga más fácil la composición đe cstas disciplinas). La historia de la física puede ayudar a que los estudiantes vayan familiarizándose con estos conceptos. El ejemplo que yo sugiero cn este scntido es el del papel que desempentó la noción de simetría en las consideraciones que llevaron a Einstein a formular la relatividad especial. Nada me. jor para ello que utilizar, como una especie de material para comentario de textos, los primeros párrafos dcl célebre artículo de Einstein de 1905, «Sobre la electrodinámica de los cuerpos en movimiento»(21).

\section{Escribia alif Einstein:}

«Es sabido que la electrodinámica de Maxwell -tal y como se entiende actualmente-conduce a asimetrias que no parecen inherentes a los fenómenos, cuando se la aplica a cuerpos en movimientos. Tómese, por ejem. plo, la acción electromagnética reciproca entre un imán y un conductor. El fenómeno que aquí se observa depende únicamente del movimiento relativo entre el con. ductor y el imán, mientras que la visión habitual establece una aguda distinción entre los dos casos en que uno u otro de estos cuerpos está en movimiento. Ya que si el imán está en movimiento y el conductor en reposo, entonces aparece en los alrededores del imán un campo eléctrico con una cierta energia definida, que produce una corriente en aquellos lugares donde se en. cuentran partes del conductor. Pero si el imán está estacionario y el conductor en movimiento, no surge nin. gún campo eléctrico en los alrededores del imán. Sin embargo, en el conductor encontramos una fuerza electromotiz, para la que no existe la energía correspondiente, pero que da lugar - suponiendo que el movi. miento relativo es el mismo en los dos casos discutidos- a corrientes eléctricas del mismo camino e intensidad que las producidas por las fuerzas eléctri. cas en el caso anterior».

«Ejemplos de este tipo»-continuaba diciendo Einstein - «junto a los intentos que sin éxito se han realizado para descubrir cualquier movimiento de la fuerza con respecto al "medio de la luz» sugieren que los fenómenos de la electrodinámica lo mismo que los de la mecánica no poseen propiedades que correspon. den a la idea de reposo absoluto».

La creencia de Einstein de que las teorias físicas no de. ben contener asimetrías formales se manifiesta en las líneas anteriores con toda claridad, y esta transparencia unida a los fenomenos físicos elementales que utiliza, así como a la «magia» que el nombre de Einstein tiene para todo típo de público, impacto que le será de gran utilidad en sus futuros estudios de física.

(d) El descubrimiento del principio de conservación de la energía

De todos es conocido el importante papel del princi- pio de conservación de la energía en la física. Su formulación general por Hermann von Helmholtz en 1847 puso a disposición de los físicos una herramienta de cuyas ventajas todavia disfrutamos. Es, por tanto, un tema a incluir, en una u otra forma, en cualquier texto de física general. Pero, junto a su enunciación precisa, seria conveniente añadir una discusión de la historia, parcial, de su descubrimiento. En particular yo sugeriría que se hiciera hincapié en la personalidad de dos de sus principales descubridores: Julius Robert Mayer y Helmholtz. ¿Por qué precisamente en ellos y no en otros como Joule, William Bhomson o Boltzmann? se pueden preguntar Vds. La razón estriba en que para estos retazos de uhistoria dirigida» (dirigida a facilitar su tarea a los alumnos de ciencias) que estoy promulgando, Mayer y Helmholtz se pueden utilizar con un fin muy concreto: el de resaltar la interconexión entre diferentes aspectos, orgánicos e inorgánicos, de la naturaleza.

Mayer y Helmholtz eran, como se sabe, médicos de formación; para cl primero la pista que le llevó a la enun. ciación del principio de conservación de la energia fue la observación de que en los trópicos la sangre de las venas cambia de color, se vuelve más roja que, por ejemplo, en Europa. (El adscribió la diferencia a la ma* yor cantidad de oxigeno presente en la sangre venosa bajo condiciones tropicales; el exceso de oxigeno cra debido a una disminución de la combustión de la comida que suministra calor al cuerpo - disminuye porque ese calor es menos necesario). En cuanto a Helmholtz basta con señalar que pertenecía a un grupo de fisiologos, du Bois-Reymond, Brüdke, Ludwig, Virchow, que se oponian al entonces todavia vigoroso planteamiento vitalista. En su célebre memoria, «Sobre la conservación de la fuerzan, el joven médico Helmholtz combinaba física y matemáticas con un cierto número de procesos biológicos. De hecho en el «esbozo autobiográfico" que escribió en 1891, inđicaba que «en la conservación de la fuerza» mi intención fue meramente proporcionar un examen crítico de dos cues. tiones: ¿Qué relaciones deben existir entre las diversas fuerzas materiales para que sea posible el movimiento perpetuo? Y ¿existen estas relaciones en la práctica y presentar los hechos para beneficio de los fisiólogos».

A! ver con este ejemplo que medicina y física se pueden fecundar mutuamente, no sólo se aprende a com. prender la unidad subyacente tras diversas ramas de la ciencia, sino que también se contribuye a combatir lo que en mi opinión es el principal abuso de la historía de la física, y de la física de hecho, en la enser̂anza. Expliqué antes las razones por las que la física se debe considerar -especialmente durante nuestro siglo- una de las ciencias más prominentes, sino la que más. Ahora bien, esta prominencia no se debe entender en un sentido excesivamente exclusivo. La riqueza conceptual, de contenidos, metodologia e historia (en un sentido socioeconomico), que aportan la química, la matemática, la geología, o las diversas ciencias bio. 
médicas no puede ser pasada por alto en modo alguno y ejemplos en los que se ve cómo incluso avances en física pueden venir de esas "otras ciencias» -el caso del principio de conservación de la energía en partedesempeñan un papel importantisimo en la ensenanza.

Antes de abandonar este tema, insistiré un instante en una de las implicaciones que tiene una excesiva prepoderancia de la física en el conjunto de las ciencias naturales. Se trata de una implicación de orden metodológica. Desde que la polémica entre Newton y Leibniz, representada por la correspondencia que intercambiaron entre 1715 y 1716 este último y Samuel Clarke, se decantara - por la fuerza de los hechos (teorías newtonianas) -en favor del Lucasian profesor del Trinity College de Cambridge, el método de la ciencia ha sido el método de la física. Naturalmente, no cabe lamentarse de este hecho, puesto que sólo beneficios nos ha reportado, pero ¿quién sabe lo que nos deparará el futuro? Piénsese, por ejemplo, en las diferencias existentes entre la medicina occidental y la oriental, más analítica, mecanicista, aquella, más sintética, globalizante la segunda; ino expresan acaso - como ya apuntó Paul Feyerabend- una indudable diferencia metodológica? Y, ya en esta línea, ¿es acaso difícil imaginar situaciones en las que las ciencias médicas no se diferencian, metodológicamente hablando, substancialmente de la física? Por supuesto todo esto son especulaciones y la especulación incontrolada es un elemento peligroso en la enseñanza, especialmente en la básica, pero también es importante educar fomentando la pluralidad de ideas $o$, mejor, de posibilidades, y en este sentido ejemplos historicos como el del principio de la conservación de la energia pueden ser extremadamente beneficiosos.

\section{(e) De Newton al átomo de Rutherford-Bohr, pasan- do por la astrofísica.}

Como último ejemplo de caso histórico a utilizar en la enser̃anza de la física en el bachillerato voy a referirme, esquemáticamente, a uno que puede cumplir diversas funciones, dos en particular: facilitar la comprension de unos temas que requieren unos conocimientos algo más avanzados de física y que, no obstante, son muy importantes para entender la posición alcanzada por la física durante el presente siglo, y resaltar la unidad e interconexión existente entre, en este caso, las diversas ramas de la física.

El ejemplo histórico en cuestión comenzaría tratando los experimentos ópticos realizados por Newton con prismas y que le llevaron a descubrir la estructura compuesta de la iuz blanca y la «elementalidad» de los que podemos denominar colores básicos. A continuación, en un gran salto temporal, se pasaria a discutir los descubrimientos espectrales realizados durante el siglo XIX (Fraunhofer, Kirchhoff, Bunsen, Balmer), en particular la construcción de espectroscopios, espectro solar y espectros de elementos y regularidades espectra- les descubiertos (hidrógeno). Con este bagaje se estaría en posición de explicar cómo la astronomia se pudo, por fin, convertirse en astrofísica. Los espectros obtenidos para todo tipo de agrupaciones estelares permitieron comenzar a averiguar su composición y, eventualmente, ya en nuestro siglo, su movimiento. Finalmente, y después de explicar los rudimentos del modeto de Rutheford, se explicaría cómo Niels Bohr liego a explicar las regularidades espectrales descubiertas por Balmer en el espectro del hidrógeno, uniendo de esta manera, en cierto sentido, óptica, astrofísica y microfísica.

\section{HISTORIA DE LA CIENCIA Y ENSEÑAN- ZA UNIVERSITARIA}

Para discutir el posible papel de la historia de la ciencia en la enseñanza universitaria es preciso distinguir entre carreras de ciencias y carreras de letras. Comenzaré por estas últimas.

Considero que la historia de la ciencia, entendida tanto desde un punto de vista internalista como externalista es absolutamente imprescindible dentro de los programas de las facultades de filosofía. Basta recordar la importancia que ha tenido durante el presente siglo la filosofía de la ciencia para la propia filosofia, para que no sea necesario entrar en argumentos más detallados (que tampoco corresponden a esta ocasión). En los estudios de historia, economía, sociologia, política $\mathrm{e}$ incluso psicologia, cursos de historia de la ciencia en los que predomine - salvo en el caso de psicologíael enfoque socioeconomico del que hablé con anterioridad son, asimismo, necesarios. Las razones en que baso mi opinión son las mismas que utilicé cuando me referí a la necesidad de introducir la historia de la ciencia en la «historia general». En el caso de la psicología la historia de la ciencia a enseñar sería una en la que se seleccionasen aquellos temas más estrechamente relacionados con ella. (En lo referente a la historia de la física, puntos claramente relacionados son aquellos conectados con la evolución de las ideas de percepción, Fechmer, Helmholtz y Mach, por ejemplo).

Paso ahora a las facultades de ciencias(22). Aquí la situación es completamente diferente a la del bachillerato. La enseñanza universitaria ha de ser, en primer lugar, rigurosa y, si se introducen cursos de historia de la ciencia, el contenido de éstos debe ser las descrip. ciones más precisas y autorizadas que un historiador de la ciencia pueda ofrecer de la manera en que se realizó un descubrimiento o desarrollada y aceptada o rechazada una teoría por científicos. Ahora bien, el problema es que estas reconstrucciones demuestran ser en general notablemente complicadas, y que para seguirlas es imprescindible aprender técnicas, conceptos, teorías o modos de proceder que en múltiples ocasiones ya están obsoletos. ¿Cuál puede ser, desde esta pers. pectiva, el beneficio que pueden extraer los aprendices 
de científicos de la historia de la ciencia? Mach, un fisico que dedicó un tiempo considerable a estudios históricos, escribió en su famoso libro Desarrollo histórico-crítico de la mecánica(23) que:

«Aquellos que conocen el curso completo del desarrollo de la ciencia podrán, como una consecuencia inmediata, juzgar más libremente y más correctamente el significado de cualquier movimiento actual que aquellos que, limitados en sus puntos de vista a la época en que transcurren sus propias vidas, contemplan meramente la tendencia momentánea que el curso de los sucesos intelectuales toma en el instante presente».

El propio ejemplo de Mach, con sus errores, juicios y valoraciones accrca de las ideas atomistas en la física que con gran celebridad desbancaron a su «análisis de las sensaciones», demuestra cuán optimistas y triviales son este tipo de argumentos.

En otras ocasiones se esgrime en favor de la historia de la ciencia el que permite «clasificar conceptos». Recientemente John Roche escribia(24):

«La mayor esperanza para descubrir y corregir errores, deslices y obscuridades en la macrofísica moderna, reside en juntar un análisis detallado de la literatura fundacional de un tema dado con una comprensión completa de la teoria moderna. El contraste con concepciones e interpretaciones anteriores nos hace conscientes de nuestras propias presuposiciones y nos ayuda a identificar y localizar la fuente de defectos de la teoría moderna. Puede incluso sugerir caminos para eliminarlos.

La historia puede también hacernos conscientes, y permitir liberarnos de los diversos fragmentos de sistemas filosóficos pasados y presentes que abundan en la física moderna, y que en ocasiones imponen restricciones en la búsqueda de explicaciones satisfactorias. La historia puede, por consiguiente, desempeñar un papel clinico en la física modernass.

Atractivas ideas, qué duda cabe, pero ¿se pueden ofrecer muchos ejemplos en su defensa? El programa de Roche es, en el mayor de los casos, un proyecto todavia por realizar y en el que yo no creo demasiado. Es cierto que en alguna ocasión puede dar resultado. Un investigador acaso se libre de atavismos y encuentre nuevas soluciones escarbando en la historia de sus disciplinas, pero iquién lo puede decir? y ¿cuánto esfuerzo habrá que dedicar a tal tarea para muy probablemente no llegar a nada? $Y$ es que las teorías científicas cambian dramáticamente: ¿qué queda del flogisto en la quimica moderna o de los modelos mecanicistas del campo electromagnético en los que incluso el propio Maxwell se empeñó?

No parece, por tanto, evidente que la historia de la ciencia ayude de manera importante a que el estudiante universitario -o investigador incluso- comprenda mejor su disciplina, pero es que además puede llegar a ser perjudicial, «subversiva» como la denominó Stephen $G$. Brush en un importante artículo publicado hace ya más de diez años y en el que discutía algunas de estas cuestiones $^{(25)}$. La manera en que se comportan - según los historiadores- los cientificos puede no ser un buen modelo para los estudiantes; los conceptos, teorías y métodos de los que se ocupa la historia de la ciencia (salvo la historia de la ciencia más reciente) pueden hacer dudar a los jóvenes universitarios, o por lo menos hacerles encontrar dificultades con las teorías en boga, y, no se olvide, que la misión principal de las facultades universitarias es formar profesionales con un conocimiento adecuado del estado actual de sus disciplinas. Hay que fomentar la creatividad, la originalidad, la duda metódica pero nunca a costa del contenido de la propia disciplina. Nos encontramos, en definitiva, con situaciones como las que describia Thomas Kuhn en su Estructura de las revoluciones cientificas. La ciencia es, sobre todo, «ciencia normal», resolver rompecabezas científicos; la revolución, el cambio de paradigma, es la excepción, lo anómalo.

A pesar de lo que he venido señalando, es posible encontrar un lugar para la historia de la ciencia en las Facultades de Ciencias. En los dos últimos cursos de carrera o como cursos de doctorado, como asignaturas optativas en cualquier caso, la historia de las distintas ciencias pueden y deben existir. Para entonces los estudiantes han obtenido una visión relativamente sólida de sus disciplinas y los riesgos que señalé antes son menos peligrosos. Además, los alumnos ya disponen más que suficientes conocimientos técnicos como para que el profesor pueda, por ejemplo, explicar la historia de la mecánica cuántica sin caer en simplificaciones que desvirtúen cualquier posible reconstrucción. Finalmente, estos cursos cumplen la función de ser lugares particularmente apropiados para que se formen los futuros historiadores de la ciencia o profesores de historia de la ciencia que toda sociedad moderna necesita.

\section{LA HISTORIA DE LA CIENCIA EN LAS ES- CUELAS NORMALES}

No quiero finalizar mi exposición sin referirme, brevemente, a las escuelas universitarias de magisterio. En realidad poco hay que decir que no venga implicado por lo que ya he señalado. He defendido la utilización de la historia de la ciencia en la enseñanza subuniversitaria y, consistentemente, hay que preparar a sus profesores para que puedan dictar las asignaturas correspondientes. Y no sólo es un deber profesional, al igual que sus futuros alumnos, los también futuros maestros, ampliarán considerablemente su visión del mundo con semejante formación. Naturalmente, la educación que las escuelas normales deben brindar ha de ser adecuada a los objetivos que se persiguen en los niveles de enseñanza en los que han de actuar los estudiantes de ma- 
gisterio, y a alguno de los cuales me referí con anterioridad. No tengo que decir, por supuesto, ante la presente audiencia que estos diseños y contenidos educativos están aún por elaborar definitivamente.

\section{NOTAS}

(1) He descrito algunos de estos desarrollos (para la historia de la fisica) asi como ciertas razones que explican el auge que ha experimentado esta disciplina en «Introducción' a Paul Forman, Cultura en Weimar, causalidad y teoria cuántica, 1918-1927, págs. 9-34 (Alianza, Madrid 1984) y "Autobiografias en física», Revista de Occidente, pp. 129-139 (Julio-Agosto 1937).

(2) Ver, por ejemplo, el discurso de Norman Lockyer, editor de Nature como presidente de la British Association for the Advancement of Science en 1903 (Southport), Report of the BAAS 1903 (John Murray, Londres 1904), pp. 3-28, o los comentarios de D.S.L. Cardwell en The organisation of science in England (Heinemann, Londres 1972), pp. 202-203.

(3) Ver en este sentido Martha Ornstein, The rôle of scientific societies in the seventeenth century (University of Chicago Press, Chicago 1928, reimpreso por Amo Press, Nueva York 1975).

(4) Una obra que puede ayudar al profesor en este terna es la de Robert K. Merton, Ciencia, fecnología y sociedad en la Inglaterra del siglo XVII (Alianza, Madrid 1984).

(5) 2 vois. (Penínsuja, Barceiona 1968).

(6) Prentice-Hall, Englewood Cliffs, New Jersey 1971.

(7) The MacMillan Press, Frome, Somerset 1983.

(8) Ver nota 4.

(9) Pengrin, Middlesex 1970.

(10) Adóison-Wesiey, Reading, Mass. 1972.

(11) Alianza, Madrid.

(12) Salvat, Pamplona 1987.

(13) The Johns Hopkins University Press, Baltimore 1986.
(14) Yale University Press, New Haven 1977.

(15) Peter Lang, Nueva York 1985.

(16) S. Shapin, "A cause in the Social Iristory of Science" Social Studies of Science 10, 231-258 (1980).

(17) «Enseñanza de las ciencias naturales por descubrimiento" Cuadernos de Pedagogia.

(18) Un articulo particularmente instructivo para algunas de estas cuestiones es el de Carlos Solis, «Astros y zanahorias" Arbor 120, 35-57 (Enero 1985).

(19) I.B. Cohen, «El descubrimiento newtoniano de la gravitación universaly Investigación y Ciencia, pp. 110-120 (Mayo 1981), reimpreso en Newton, pp. $19-46$ (Conscjo Nacional de Ciencia y Tecnologia, México 1982).

(20) Fondo de Cultura Económica, México 1965.

(21) He discutido este tema en el capitulo 4 de mi obra $E l$ origen y desarrollo de la relatividad (Alianza, Madrid 1935).

(22) Los problemas asociados con la posible utilización de la historia de la ciencia en las escuejas técnicas requerirían un tratamiento específico en el que no puedo entrar aquí. Un clemento que distingue y complica la situación con respecto a las facultades de ciencias, es la historia de ia tecnologia a la que, evidentemente, habria que tomar en consideración.

(23) E. Mach.

(24) J. Roche, «History as surgery» Phys. Bull. 35, 414-415 (1984).

(25) S.G. Brush, «Should the History of Science be Rated $\mathrm{X}$ ?", Science 183, 1164-1172 (1974). 